\title{
The relationship between maternal body fat and pre-implantation embryonic weight: Implications for survival and long-term development in an assisted reproductive environment ${ }^{*}$
}

\author{
Julie Weathers ${ }^{1}$, Natalie Zimmerer ${ }^{1}$, Lindsay Penrose ${ }^{2}$, Kory Graves-Evenson ${ }^{1}$, Samuel Prien ${ }^{1,2}$ \\ ${ }^{1}$ Department of Animal and Food Sciences, Texas Tech University, Lubbock, USA \\ ${ }^{2}$ Department of Obstetrics and Gynecology, Texas Tech University Health Sciences Center, Lubbock, USA \\ Email: samuel.prien@ttuhsc.edu \\ Received 18 May 2013; revised 20 June 2013; accepted 28 June 2013 \\ Copyright (c) 2013 Julie Weathers et al. This is an open access article distributed under the Creative Commons Attribution License, \\ which permits unrestricted use, distribution, and reproduction in any medium, provided the original work is properly cited.
}

\begin{abstract}
There can be little argument that embryo quality is one of the most critical factors in the success of assisted reproductive techniques. Yet the current methods of grading embryos are subjective at best. While a number of different groups have described more qualitative means of assessing embryo quality, the current standard remains morphology. Morphology has proven a good standard, but it does not allow for the detection of chromosomal abnormalities nor can it assess the biochemical status of the embryo prior to transfer. This laboratory recently described a method to estimate embryo weight and suggested weight might be a good indicator of biochemical status. The objective of the present study was to determine the relationship between maternal body composition and embryo weight and determine the influence maternal chemistry had on embryo development. The data continue to suggest that maternal body composition, especially body fat, influences the chemical nature of the embryo and may play a critical role in long-term survival.
\end{abstract}

Keywords: Embryo Weight; Body Fat; Assisted Reproductive Technologies; Specific Gravity

\section{INTRODUCTION}

It is well documented that most assisted reproductive techniques (ARTs) carry significant risk of multiple gestations [1-3]. A recent report has linked ART and its associated multigestational outcomes not only with increased infant

"Presented in part at the 2010 and 2011 Annual Meetings of ASRM. risks, but with significantly increasing the cost of health care [4]. While in principle it is generally agreed the solution to this issue in to establish single embryo transfer (SET) as the norm, there is currently no way to reliably assess the developmental potential of pre-implantation embryos conceived via ART.

Currently most programs rely on a combined system of embryo morphology and development rates in culture to predict success in vivo [5]. While the system has increased pregnancy rates [6], it has not proven reliable enough on its own to move totally to SET, This is due, at least in part, to the fact that early stage embryos with aneuploidy can develop in a normal fashion and have normal appearing morphology [7].

Recently, a number of new approaches have been proposed to provide a non-invasive means of evaluating embryo quality. These would include, measure of various metabolites [8-10], glucose utilization [11,12], and a number of proteomic methodologies [13]. While these methods hold great promise, to date no fully reliable method has been developed utilize these methods in clinical embryo assessment.

Previous research has suggested that embryo viability might be influenced by the chemical constituents of the embryo [14-16]. Work from this laboratory has suggested that embryo weight is at least in part a function of its chemical composition and that because of the routine shape and size of early stage embryos these differences can be determined using a simple modified specific gravity technique (SGT) [17]. The object of the present study was to explore the relationship between maternal body composition, embryo weight and embryo survival with the longterm goals of assessing how embryo weight (composition) might influence embryo develop and survival, and how this 
might influence pregnancy rates and cryopreservation.

\section{MATERIAL AND METHODS}

\subsection{Acquisition of Embryos}

Using IACUC approved protocols, three separate experiments were conducted to determine the influence of maternal body composition on embryo weight. In the first experiment, embryos were obtained from 6 - 8 weeks old mice from a wild-type CF6F1 mouse (control), a C57BL/6NCrl-Lepr ${ }^{\mathrm{db}-\mathrm{lb}} / \mathrm{Crl}$ mouse or "Pound Mice ${ }^{\mathrm{tm} \text { " }}$ (Charles Rivers; Burlington, MA) or its progenitor the C57BL/6NCrl. The C57BL/6NCrl-Lepr ${ }^{\mathrm{db}-\mathrm{lb}} / \mathrm{Crl}$, mice that are homozygous for the mutation lack receptors for leptin, and can become severely obese, particularly when fed a specific diet. However, mice with a single copy of the gene remain relatively lean under similar conditions, Therefore there were three embryo groups: the wild-type control, the heterozygous lean C57BL/ $6 \mathrm{NCrl}$ (lean), and the obese C57BL/6NCrl-Lepr ${ }^{\mathrm{db}-\mathrm{lb}} / \mathrm{Crl}$ (obese). In the second experiment, embryos were obtained from only the C57BL/6NCrl or C57BL/6NCrl$\mathrm{Lepr}^{\mathrm{db}-\mathrm{lb}} / \mathrm{Crl}$ animals. The mice were either lean or obese. In the final experiment, embryos were obtained from the obese mice with average body weights of 30 , 40 or 50 g respective.

Prior to collection of embryos, all mice were injected with $5 \mathrm{mIU}$ of pregnant mare serum gonadotropin (Sigma-Aldrich; St. Loius, MO), followed 24 h later by 5 mIU hCG (Merck, Whitehouse Station, NJ) and mating. As these experiments were to examine the maternal contribution to embryo chemical composition, all females were mated to wild-type males. This prevented physical issues with obese types mating and, according to the supplier, represents the first successful in vitro recover of embryos from the obese animals. The animals were weighed and euthanized approximately $24 \mathrm{~h}$ after mating in hopes of obtaining 1 or 2 cell embryos. Once euthanized the oviducts were excised and placed in sterile media in $65 \mathrm{~mm}$ culture dishes (Falcon; Becton Dickinson; Franklin Lakes, NJ). In the case of the obese animals this was a two-step process. Once the mouse peritoneum was opened, the ovary and uterine horn were identified and all tissue, including significant adipose tissue was removed. Working under a dissecting microscope (Nikon SMZ-2, Nikon Inc; NY), the adipose tissue was teased apart to expose the oviducts which were significantly distorted. The oviducts were then transferred to a second dish to excise the embryos. Once identified embryos were transferred to organ culture dishes with fresh Ham's F-10 with $10 \%$ serum substitute supplement (Irvine Scientific; Santa Ana, CA).

\subsection{Experimental Specific Gravity Device}

In general specific gravity is a non-specific measurement of density; however, as weight is a function of mass and mass is a function of density, if shape is controlled (such as a shape of an embryo), specific gravity can be used to estimate weight as a function of mass. Therefore, a chamber was constructed that allowed the visualization of embryos and embryo shaped substances of known weight as they "dropped" a known distance. Prior to dropping embryos through the chamber, borosilicate and barium-titanate beads (MO-SCI Specialty Products, L.L.C.; Rolla, MO) of known weight and similar in size and shape to the mice embryos were dropped through the device and their drop time to weight ratios used to establish a standard curve.

\subsection{Experiment 1}

In experiment \#1 embryos were collected from 11 mice (4 control, 4 lean, and 3 obese-a fourth animal was attempted but had no embryos). The mice were weighted just prior to euthanasia and demonstrated average weights of 17.9, 22.9 and $49.9 \mathrm{~g}$ for the control, lean and obese mice respectively. One and two cell embryo weights were then estimated by passing embryos through the specific gravity chamber. In this initial experiment once embryos were weighed they were discarded.

\subsection{Experiment 2}

As experiment \#1 demonstrated similar estimated weights for the control and lean mice, experiment \#2 compared weights for mice of similar genetics (C57BL/ 6NCrl and C57BL/6NCrl-Lepr ${ }^{\mathrm{db}-\mathrm{lb}} / \mathrm{Crl}$ ) over time. Embryos were obtained from 7 mice, (3 lean and 4 obese). Once collected the embryos were measured using the SGT to estimate their weight and then returned to culture. The embryos were reweighed and assessed for cell number on days 2, 4 and 6 to determine changes in weigh due to increases in cell numbers.

\subsection{Experiment 3}

In the final experiment, embryo weights were estimated in animals of similar genetics across the weight range established in earlier experiments. The C57BL/6NCrl$\mathrm{Lepr}^{\mathrm{db}-\mathrm{lb}} / \mathrm{Crl}$ mice were mated as they had average weights of 30, 40 or 50 grams (5 mice per weight group). Day one embryo weights were then estimated using the SGT.

\subsection{Statistical Analysis}

Initial estimated embryo weights were compared to material weights using analysis of variance and Tukey's 
mean separation test using the Statistical Package for the Social Sciences (Version 12; Chicago, IL). To remove variability due to operator error of the timer, all data underwent negative logarithmic transformation prior to analysis. Data for continued culture in experiment \#2 were compared using a two way analysis of variance by embryo source and time.

\section{RESULTS}

One-hundred of each of the various weights of borosilicate and barium-titanate beads where dropped through the system to establish the standard curve. Descent times were amazingly similar between beads of the same weight and size with an overall inner-assay C.V. of 4.1\%. As the borosilicate beads were closer in size to the actual mice embryos, their data were then used to calculate the final curve. Using the points established by the beads, the curve was completed by extrapolating points for zero time and zero weight mathematically and resulted in the formula:

$$
y=0.4216 \operatorname{Ln}(x)+2.6334
$$

where $x$ represented the descent time of the bead/embryo and $y$ the resultant estimated weight. As expected, the relationship between weight (density) and time of descent was not linear but curvilinear on a logarithmic scale (Figure 1). This formula, Eq.1, was then used to estimate weights in all further experiments.

As designed, there were significant differences in the average weights of the mice due to either strain or feed status $(\mathrm{P}<0.001)$. The control mice in experiment \#1 averaged $17.9 \mathrm{~g} \pm 1.2 \mathrm{~g}$ STD, significantly lower than the obese mice $(49.9 \mathrm{~g} \pm 5.8 \mathrm{~g}$ ) but similar in weight to the lean animals (22.9 $\mathrm{g} \pm 2.2 \mathrm{~g}$ ). Visual observation of the peritoneal cavity at the time of embryo retrieval confirmed the increased weight in the obese mice was due to lipid storage in the form of adipose tissue; in many cases requiring the modified technique described in the materials and methods to locate the oviduct. As suggested from a previous study using the SGT and confirmed by transmission electron microscopy in that study [17], there was an overall decrease in estimated initial embryo weight (one or two cell) associated with the drastic increase in maternal weight due to the adipose tissue seen in the obese mice (Figure 2). This observation appeared to be confirmed by the similar maternal weights and their associated similar estimation of embryo weights between the controls and the lean animals. Further, a second experiment comparing only the lean and obese mice demonstrated similar results Figure 3).

In experiment \#2 it was found that while the embryos of the lean animals continue to grow and develop in a

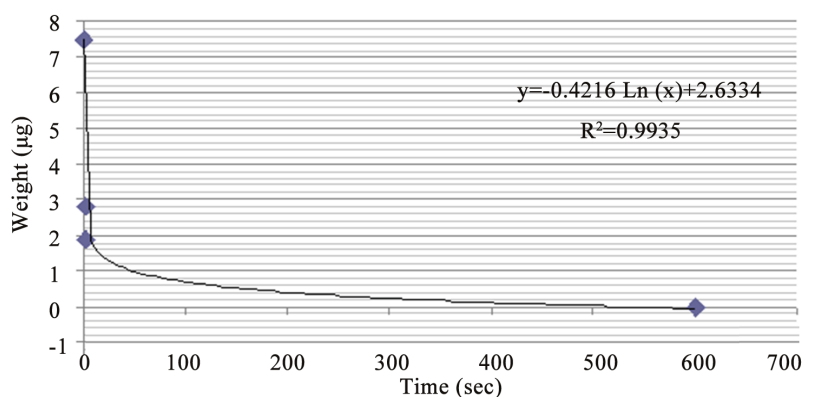

Figure 1. Development of a standard curve for the estimation of early stage embryo weights using spherical borsilicant beads of similar size to mice embryos then mathematically forcing a zero weight and zero time point to establish an equation for estimation of embryo weight.

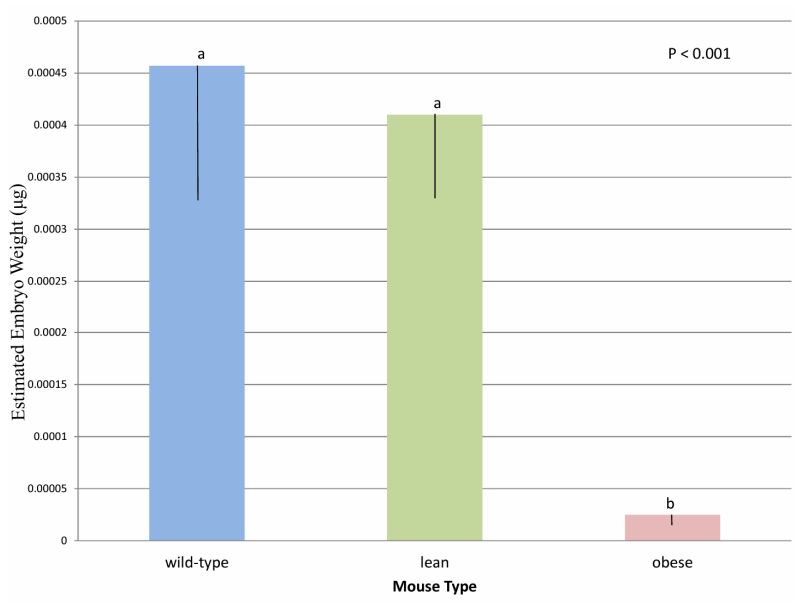

Figure 2. A comparison of one or two cell embryo weights as determined using a novel specific gravity technique. Embryo weights do not appeared to be related to the strain of mouse donating the embryo (CB6F1 verses the leptin receptor intact C57BL/6NCrl in animals of the same approximate weight but do appear to be related to body condition of the donor (lean C57BL/6NCrl verses the obese C57BL/6NCrl-Lepr $\left.{ }^{\mathrm{db}-\mathrm{lb}} / \mathrm{Crl}\right)$. Means with different subscripts differ at the $\mathrm{P}<0.001$ level.

normal pattern, the embryos of the obese animals demonstrated significant developmental delays if not stalling out completely (Figure 4). Interestingly, the lean embryos initial demonstrated a weight loss between days 1 and 2 (possibly due to transformation from a large single cell to smaller, multicellular embryos) then a steady weight gain over the remaining culture period (Figure 3) possible due to the addition of water especially in the blastocoel cavity of latter stage embryos.

Finally, the relationship between increasing maternal weight due to the addition of adipose tissue verses decreasing estimated embryo weight appears almost linear (Figure 5). This observation might be expected from the previous study which suggested a higher incorporation of lipids throughout the embryo cellular complex. 


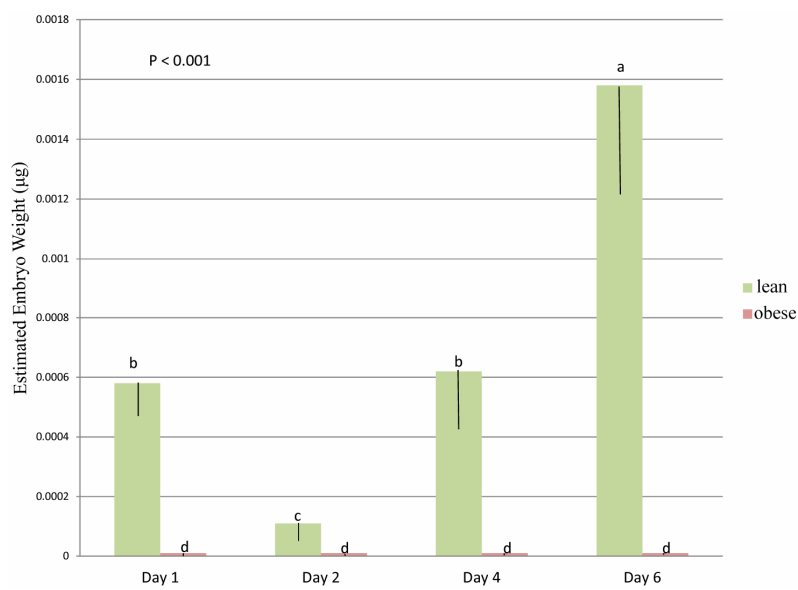

Figure 3. A comparison of estimated embryo weights from mice with lean and obese body styles over the first 6 days of development, demonstrating changes in embryo weight with cellular duplication and reconfiguration of cellular components water content. Means with different subscripts differ at the $\mathrm{P}<$ 0.001 level.

\section{DISCUSSION}

There can be little doubt that the oocyte serves as the major source of cellular components for early stage embryos. Further, it is also well established that changes in cellular chemistry can have significant impact on cellu assume that maternal body composition could have an influence on the membrane and cytoplasmic constituents of the oocyte and embryo and therefore their function.

Previous research has described the influence of growth environment on embryo development. It is well documented that abnormal levels of certain nutrients influence growth patterns [14-16,18,19] and therefore might be an important means of assessing embryo quality in the field of assisted reproductive technologies [8-13].

The present study describes a novel approach to assess membrane constituents, specific gravity. Because oocytes and early stage embryos are generally of a consistent size and shape within any give specie; specific gravity, which is truly a measure of mass, should also provide a reasonable estimation of weight.

Using this estimation, it was possible to demonstrate a relationship between maternal body composition and its influence on the weight of early (one and two cell) embryos. Given previous work from this lab which confirmed that embryos conceived from cattle breeds with high body lipid content had large deposits of free lipid droplets in the embryo cellular cytoplasm [17], and the relative simple metabolic pathways present in oocytes/embryos, it can be assumed that the presence of such large amounts of any compound in the cytoplasm would have distinct influences on cellular processes and the ability to manipulate those processes during ART

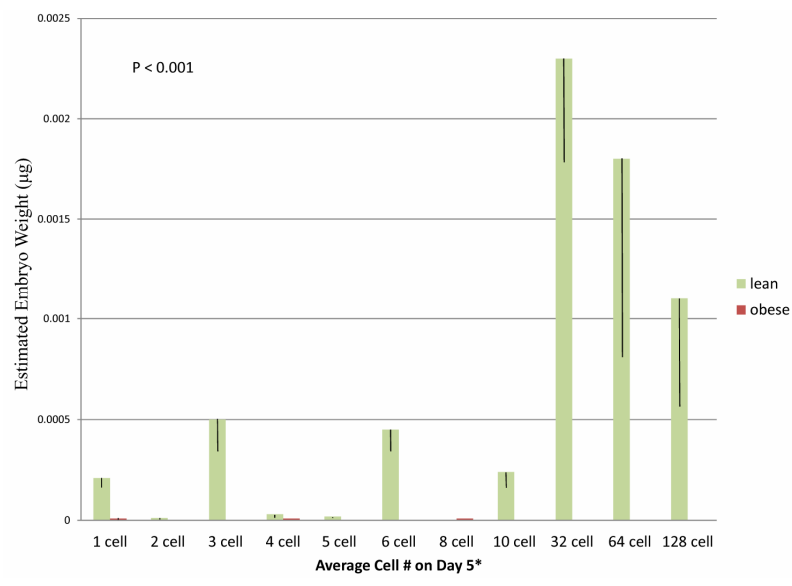

Figure 4. Estimated embryos weights from day-5 embryos with different cell \# obtained from mice with lean and obese body styles. Later stage embryos were assigned cells \# of: morula-32, early blastocyst-64 and later blastocyst-128, for statistical analysis.

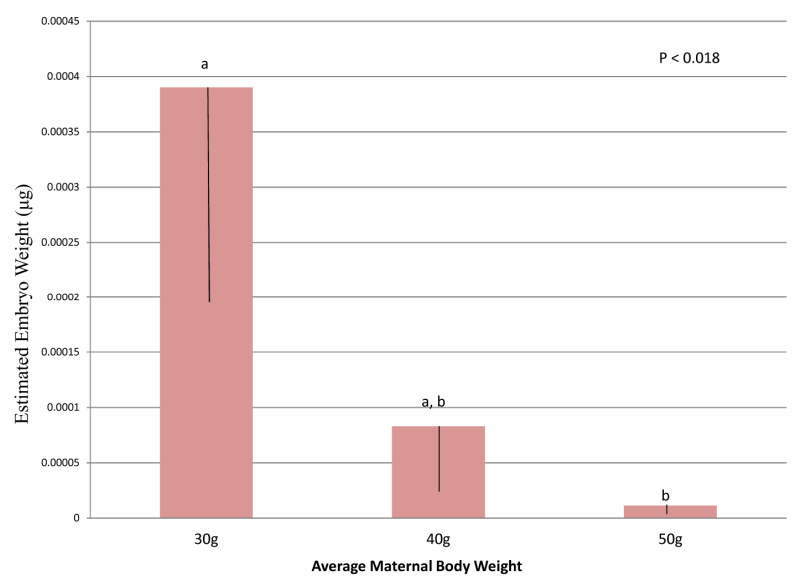

Figure 5. A comparison of estimated embryo weight between maternal donors lacking the leptin receptor (C57BL/6NCrlLeprdb-lb/Crl) and with increasingly obese body styles (30 g obese, $40 \mathrm{~g}$-significantly obese, $50 \mathrm{~g}$-severally obese) as estimated by specific gravity. Means with different subscripts differ at the $\mathrm{P}<0.018$ level.

procedures. This reasoning is supported in part by the poor development seen in the embryos derived from the obese animals.

The new SGT appears to be a potential tool for noninvasive assessment of embryo quality. It is important to note that if the technique is adopted each media used would need to be evaluated and a mathematical equation establish as each of these media has its own specific gravity which will influence the descent time of the embryos being tested. However, if an estimation of weight can be used as a judgment of embryo energy reserves or embryo developmental potential it might allow a more quantitative assessment of embryo quality and increase the chance of success from SET. Alternatively, being able to determine an embryo's constituent 
makeup might allow adjustment to cryoprotectants, lessening the stresses being put on embryos during cryopreservation and increasing embryo survival rates.

Studies are currently underway with a redesigned SGT chamber which ensures embryo recovery, to assess influence of the chamber on embryo development and to determine if there is a relationship between the estimated weight of one-cell embryo (most consistent shape) within a single collected pool of embryos and their eventual ability to develop to the blastocyst stage and establish pregnancy.

\section{ACKNOWLEDGEMENTS}

The authors wish to acknowledge and thank the American Jersey Cattle Association and the Laura Bush Women's Health Research Institute for grant support of these studies.

\section{REFERENCES}

[1] Templeton, A. and Morris, J.K. (1998) Reducing the risk of multiple births by transfer of two embryos after in vitro fertilization. The New England Journal of Medicine, 339, 573-577. doi:10.1056/NEJM199808273390901

[2] Templeton, A. (2000) Assessing the outcome of IVF. Annals of the New York Academy of Sciences, 900, 345350. doi:10.1111/j.1749-6632.2000.tb06247.x

[3] Sullivan, E.A., Zegers-Hochschild, F., Mansour, R., Ishihara, O., de Mouzon, J., Nygren, K.G. and Adamson, G.D. (2013) International Committee for Monitoring Assisted Reproductive Technologies (ICMART) world report: Assisted reproductive technology 2004. Human Reproduction, 28, 1375-1390. doi:10.1093/humrep/det036

[4] Chang, H.H., Larson, J., Blencowe, H., Spong, C.Y., Howson, C.P., Cairns-Smith, S., Lackritz, E.M., Lee, S.K., Mason, E., Serazin, A.C., Walani, S., Simpson, J.L., Lawn, J.E. and Born Too Soon Preterm Prevention Analysis Group (2013) Preventing preterm births: Analysis of trends and potential reductions with interventions in 39 countries with very high human development index. Lancet, 381, 223-234. doi:10.1016/S0140-6736(12)61856-X

[5] Racowsky, C., Vernon, M., Mayer, J., Ball, G.D., Behr, B., Pomeroy, K.O., Wininger, D., Gibbons, W., Conaghan, J. and Stern, J.E. (2010) Standardization of grading embryo morphology. Journal of Assisted Reproduction and Genetics, 27, 437-439.

doi:10.1007/s10815-010-9443-2

[6] Gerris, J., De Neubourg, D., Mangelschots, K., et al. (1999) Prevention of twin pregnancy after in-vitro fertilization or intracytoplasmic sperm injection based on strict embryo criteria: A prospective randomized clinical trial. Human Reproduction, 14, 2581-2587. doi:10.1093/humrep/14.10.2581

[7] Alfarawati, S., Fragouli, E., Colls, P., et al. (2011) The relationship between blastocyst morphology, chromo- somal abnormality, and embryo gender. Fertility and Sterility, 95, 520-524. doi:10.1016/j.fertnstert.2010.04.003

[8] Lane, M. and Gardner, D.K. (1996) Selection of viable mouse blastocysts prior to transfer using a metabolic criterion. Human Reproduction, 11, 1975-1978. doi:10.1093/oxfordjournals.humrep.a019527

[9] Houghton, F.D., Hawkhead, J.A., Humpherson, P.G., et al. (2002) Non-invasive amino acid turnover predicts human embryo developmental capacity. Human Reproduction, 17, 999-1005.

[10] Brison, D.R., Houghton, F.D., Falconer, D., et al. (2004) Identification of viable embryos in IVF by non-invasive measurement of amino acid turnover. Human Reproduction, 19, 2319-2324. doi:10.1093/humrep/deh409

[11] Gardner, D.K. and Leese, H.J. (1987) Assessment of embryo viability prior to transfer by the noninvasive measurement of glucose uptake. Journal of Experimental Zoology, 242, 103-105. doi:10.1002/jez.1402420115

[12] Jones, G.M., Trounson, A., Vella, P.J., et al. (2001) Glucose metabolism of human morula and blastocyst-stage embryos and its relationship to viability after transfer. Reproductive BioMedicine, 3, 124-132

[13] Sakkas, D. and Gardner, D.K. (2005) Noninvasive methods to assess embryo quality. Current Opinion in Obstetrics and Gynecology, 17, 283-288. doi:10.1097/01.gco.0000169106.69881.3e

[14] Muñoz, G. and Bongiorni-Malavé, I. (1979) Influence of dietary protein restriction on ovulation, fertilization rates and pre-implantation embryonic development in mice. Journal of Experimental Zoology, 210, 253-257. doi:10.1002/jez.1402100208

[15] Abe, H., Yamashita, S., Satoh, T. and Hoshi, H. (2002) Accumulation of cytoplasmic lipid droplets in bovine embryos and cryotolerance of embryos developed in different culture systems using serum-free or serum-containing media. Molecular Reproduction and Development, 61, 57-66. doi:10.1002/mrd.1131

[16] Barcelo-Fimbres, M. and Seidel Jr., G.E. (2007) Effects of either glucose or fructose and metabolic regulators on bovine embryo development and lipid accumulation in vitro. Molecular Reproduction and Development, 74, 1406-1418. doi:10.1002/mrd.20700

[17] Weathers, J. (2008) Early indications of breed differences for cryopreservation of embryos in cattle. Master's Thesis. https://repositories.tdl.org/ttu-ir/handle/2346/18883

[18] Roche, J.F. (2006) The effect of nutritional management of the dairy cow on reproductive efficiency. Animal Reproduction Science, 96, 282-296. doi:10.1016/j.anireprosci.2006.08.007

[19] Luzzo, K.M., Wang, Q., Purcell, S.H., et al. (2012) High fat diet induced developmental defects in the mouse: Oocyte meiotic aneuploidy and fetal growth retardation/brain defects. 2012. http://www.ncbi.nlm.nih.gov/pubmed/23152876 